\title{
A logistic approximation to the cumulative normal distribution
}

\author{
Shannon R. Bowling ${ }^{1}$; Mohammad T. Khasawneh²; \\ Sittichai Kaewkuekool ${ }^{3}$; Byung Rae $\mathrm{Cho}^{4}$ \\ ${ }^{1}$ Old Dominion University (USA); ${ }^{2}$ State University of New York (USA); \\ ${ }^{3}$ King Mongkut's University of Technology Thonburi (THAILAND); ${ }^{4}$ Clemson University (USA) \\ sbowling@odu.edu; mkhasawn@bingbamton.edu; sittichai.kae@,kmutt.ac.th;bcho@,clemson.edu
}

Received February 2009

Accepted March 2009

\begin{abstract}
This paper develops a logistic approximation to the cumulative normal distribution. Although the literature contains a vast collection of approximate functions for the normal distribution, they are very complicated, not very accurate, or valid for only a limited range. This paper proposes an enhanced approximate function. When comparing the proposed function to other approximations studied in the literature, it can be observed that the proposed logistic approximation has a simpler functional form and that it gives higher accuracy, with the maximum error of less than 0.00014 for the entire range. This is, to the best of the authors' knowledge, the lowest level of error reported in the literature. The proposed logistic approximate function may be appealing to researchers, practitioners and educators given its functional simplicity and mathematical accuracy.
\end{abstract}

Keywords: normal distribution, logistic, approximation, minimax criteria

\section{Introduction}

The most important continuous probability distribution used in engineering and science is perhaps the normal distribution. The normal distribution reasonably describes many phenomena that occur in nature. In addition, errors in measurements are extremely well approximated with the normal distribution. In 1733, DeMoivre developed the mathematical equation of the normal curve. It 
provided a basis on which much of the theory of inductive statistics is founded. The normal distribution is often referred to as the Gaussian distribution, in honor of Karl Friedrich Gauss, who also derived its equation from a study of error in repeated measurements of an unknown quantity. The normal distribution finds numerous applications as a limiting distribution. Under certain conditions, the normal distribution provides a good approximation to binomial and hypergeometric distributions. In addition, it appears that the limiting distribution of sample averages is normal. This provides a broad base for statistical inference that proves very valuable in estimation and hypothesis testing.

If a random variable $X$ is normally distributed with mean $\mu$ and variance $\sigma^{2}$, its probability density function is defined as

$$
f(x)=\frac{1}{\sqrt{2 \pi} \sigma} \exp \left[-\frac{1}{2}\left\{\frac{x-\mu}{\sigma}\right\}^{2}\right], \quad-\infty<x<\infty
$$

Equation 1. "PDF of Normal Distribution".

From an inspection of the normal distribution by examination of its first and second derivatives, the following properties of the distribution are known:

- The mode, which is the point on the horizontal axis where the curve is a maximum, occurs at $x=\mu$.

- The curve is symmetric about a vertical axis through the mean $\mu$.

- The curve has its points of inflection at $x=\mu \pm s$.

- The curve is convex upward, concave upward, concave downward, convex downward, when $-\infty<x<\mu-\sigma, \mu-\sigma \leq x \leq \mu, \mu<x<\mu+\sigma$, and $x \geq \mu+\sigma$, respectively.

- The curve approaches the horizontal axis asymptotically as we proceed in either direction away from $\mu$.

- The total area under the curve and above the horizontal axis is equal to one.

The cumulative normal distribution is well known as 


$$
\Phi(x)=\int_{-\infty}^{x} \frac{1}{\sqrt{2 \pi} \sigma} \exp \left[-\frac{1}{2}\left\{\frac{x-\mu}{\sigma}\right\}^{2}\right] d y .
$$

Equation 2. "CDF of Normal Distribution".

The difficulty encountered in solving integrals of normal density functions necessitated the tabulation of normal curve areas for quick reference. However, it would be an enormous task to attempt to set up separate tables for every conceivable value of $\mu$ and $\sigma$. Fortunately, we are able to transform all the observations of any random variable $X$ to a new set of observations of a normal random variable $Z$ with mean zero and variance one by means of transformation $Z$ $=(x-\mu) / \sigma$. The standard normal distribution is then given by

$$
f(z)=\frac{1}{\sqrt{2 \pi}} \exp \left[-\frac{1}{2} z^{2}\right]
$$

Equation 3. "Standard Normal Distribution".

and similarly the cumulative standard normal function is defined as

$$
\Phi(z)=\int_{-\infty}^{z} \frac{1}{\sqrt{2 \pi}} \exp \left[-\frac{y^{2}}{2}\right] d y
$$

Equation 4. "Cumulative Standard Normal Distribution".

Unfortunately, there is no closed-form solution available for the above integral, and the values are usually found from the tables of the cumulative normal distribution. From a practical point of view, however, the standard normal distribution table only provides the cumulative probabilities associated with certain discrete $z$-values. When the $z$-value of interest is not available from the table, which frequently happens, practitioners often guess its probability by means of a linear interpolation using two adjacent $z$-values, or rely on statistical software.

In order to rectify this practical inconvenience, a number of approximate functions for a cumulative normal distribution have been reported in the research community. The literature review indicates, however, that they are mathematically complicated, do not have much accuracy, and lack validity when the entire range 
of $z$-values is considered. In order to address these shortcomings, this paper develops a logistic approximate function for the cumulative normal distribution. The mathematical form of the proposed function is much simpler than the majority of other approximate functions studied in the literature. In fact, probabilities can be even obtained by using a calculator. Further, the accuracy of the proposed function is higher than with the other approximate functions.

The remainder of the paper is organized as follows. In section 2, the existing literature on approximations to a cumulative normal distribution is discussed. Section 3 first discusses a logistic distribution and notes the similarities and differences between the logistic and normal distributions are noted. Section 3 then proposes the modified logistic approximate function by numerically identifying polynomial regression coefficients in such a way that the absolute maximum deviation between the cumulative distribution and the modified logistic function is minimized. Section 4 evaluates the accuracy of the proposed approximate function, and section 5 discusses and concludes about the results of this paper.

\section{Review of Prior Research}

It is often beneficial to represent the distribution functions of random variables using a convenient approximation, facilitating mathematical and statistical analysis. In particular, some families of distributions have been constructed to provide approximations of a wide variety of distributions, whenever possible. Such families are frequently called systems of distributions (see Johnson and Kotz, 1969), and the particular requirements for these systems are ease of computation and facility of algebraic manipulation, while attaining a reasonable approximation. A major criterion in judging an approximate function for a certain distribution is the use of as few parameters as possible in defining the distribution.

From the point of view of replacing the normal distribution by another distribution, the following can be observed: First, a lognormal distribution can give a good representation which has a small absolute value, say less than 0.25 , of the coefficient of variation. Second, a particular form of logistic distribution is very close to a normal distribution. Finally, a form of the Weibull distribution with the shape parameter close to 3.25 is almost identical with the standard normal distribution. In addition to the above-mentioned general approximations, a number 
of researchers attempted to find other approximations, which are summarized in Table 1.

\section{Proposed Model}

\subsection{A Logistic Distribution Revisited}

The logistic distribution is defined as

$$
f(z)=\frac{\exp [-\{z-\alpha\} / \beta]}{\beta[1+\exp \{-(z-\alpha) / \beta\}]^{2}},-\infty<x<\infty
$$

Equation 5. "Logistic Distribution".

with $E(z)=\alpha$, and $\operatorname{Var}(z)=\frac{\pi \beta^{2}}{3}=3.289868 \beta^{2}$. The cumulative logistic distribution is:

$$
F(z)=\frac{1}{1+\exp [-\{z-\alpha\} / \beta]}
$$

Equation 6. "Cumulative Logistic Distribution".

The logistic distribution has been used extensively to represent growth functions, with $z$ representing time. The use of the logistic curve for economic demographic purposes has been very popular from the end of nineteenth century onwards. The fact that a logistic distribution has a shape similar to that of the normal distribution makes it convenient to replace the normal by the logistic in order simplify the analysis in engineering and science. However, such substitutions must be done with care. Although there is a close similarity in shape between the normal and logistic distributions, two distinguishable differences are noted. First, the value of the kurtosis for the logistic is 4.2, considerably different from the value 3 for the normal distribution. The difference is attributed largely to the relatively longer tails of the logistic distribution. Second, the logistic distribution with mean zero and variance one specifies $\alpha=1$ and $\beta=0.551329$, and is symmetrical about $\alpha$ with the inflection points at $z= \pm \frac{\sqrt{3}}{\pi} \cdot \ln (2+\sqrt{3})= \pm 0.726077$, whereas the standard normal curve has the inflection points at $z= \pm 1$. 


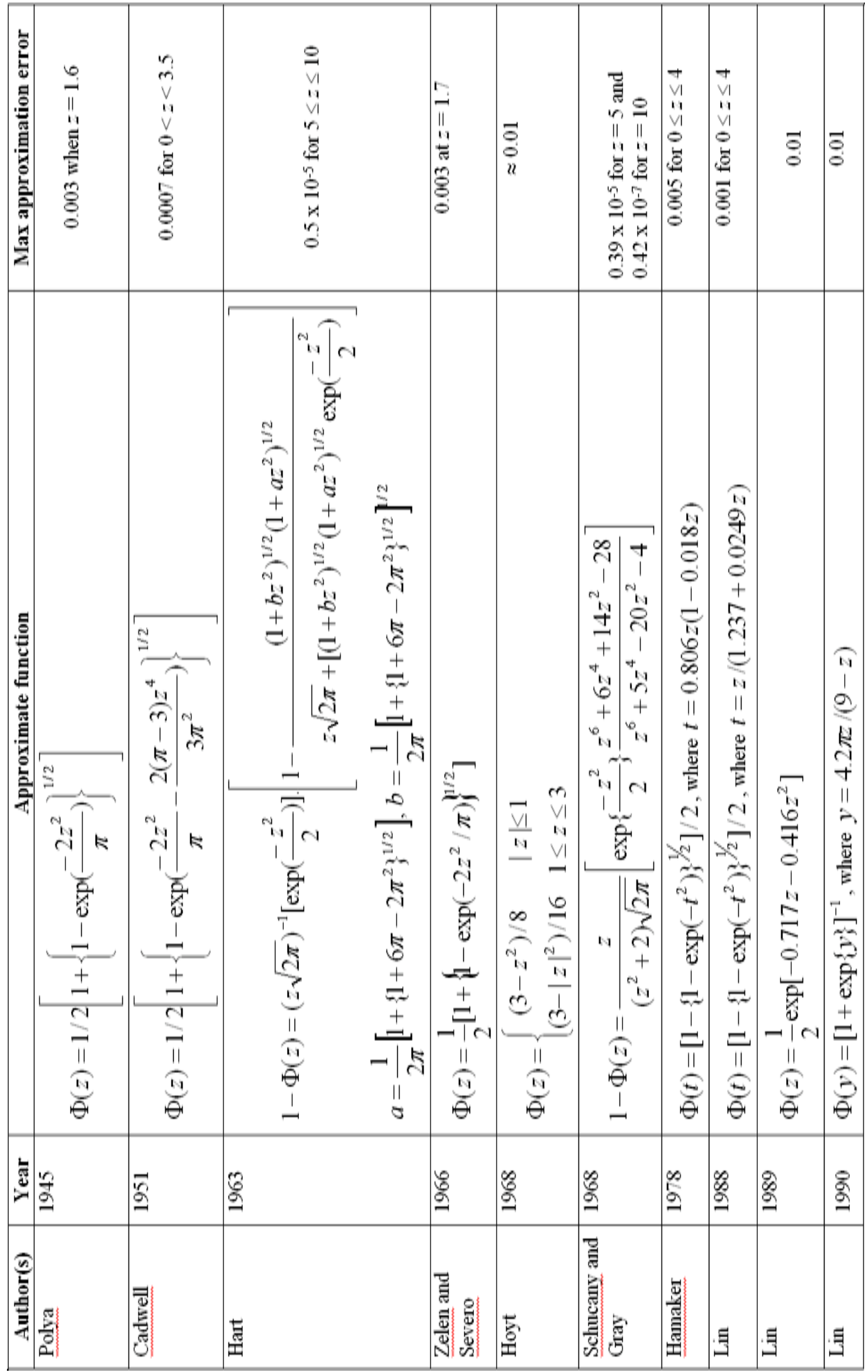

Table 1. "A summary of approximate functions of the cumulative normal distribution". 


\subsection{The Effect of $\gamma$}

The cumulative logistic distribution with mean zero and variance one is known as $[1+\exp (-\gamma z)]^{-1}$ where $\gamma=\pi / \sqrt{3}$. Simulation results indicate that the parameter coefficient $\gamma$ has a significant effect on the kurtosis and the inflection points. In order to show the effect of $\gamma$, the absolute deviation function,

$$
D(z)=\left|\int_{-\infty}^{z} \frac{1}{\sqrt{2 \pi}} \exp \left[-\frac{u^{2}}{2}\right] d u-\frac{1}{1+\exp [-\gamma z]}\right|
$$

Equation 7. "Difference between Normal and Logistic".

is considered. This absolute deviation function is a function of the absolute deviation between the cumulative standard normal distribution and the cumulative logistic distribution with mean zero and variance one. Using the generalized reduced gradient algorithm (see Hillier and Liberman, 2001), the parameter $\gamma$ is determined by minimizing the maximum deviation between the cumulative normal distribution and the logistic function. As can be seen in Figure 1, the maximum deviation of 0.0095 occurs at $z= \pm 0.57$ for $\gamma=1.702$. Therefore, the following equation gives the best logistic fit for the cumulative normal distribution,

$$
F(z) \approx \frac{1}{1+e^{-1.702 z}}
$$

Equation 8. "Best Fit One Parameter Logistic Equation".

The functional relationship between $\gamma$ and the deviation function over $-4.5 \leq z \leq 4.5$ is shown in Figure 1 . Note that the absolute deviation with $\gamma \approx 1.702$ becomes relatively small at $z \approx \pm 1.2$ and $z=0$. As shown in Figure 2 , sensitivity analysis indicates that varying the value of $\gamma$ changes the values at which the deviation function intersects the $z$-axis. Two questions remain unanswered at this point. First, how can the effect of $\gamma$ on the absolute deviations be better captured? Second, how can a consistent judgment on the degree of goodness of fit associated with $\gamma$ be made in order to minimize the absolute deviations? 


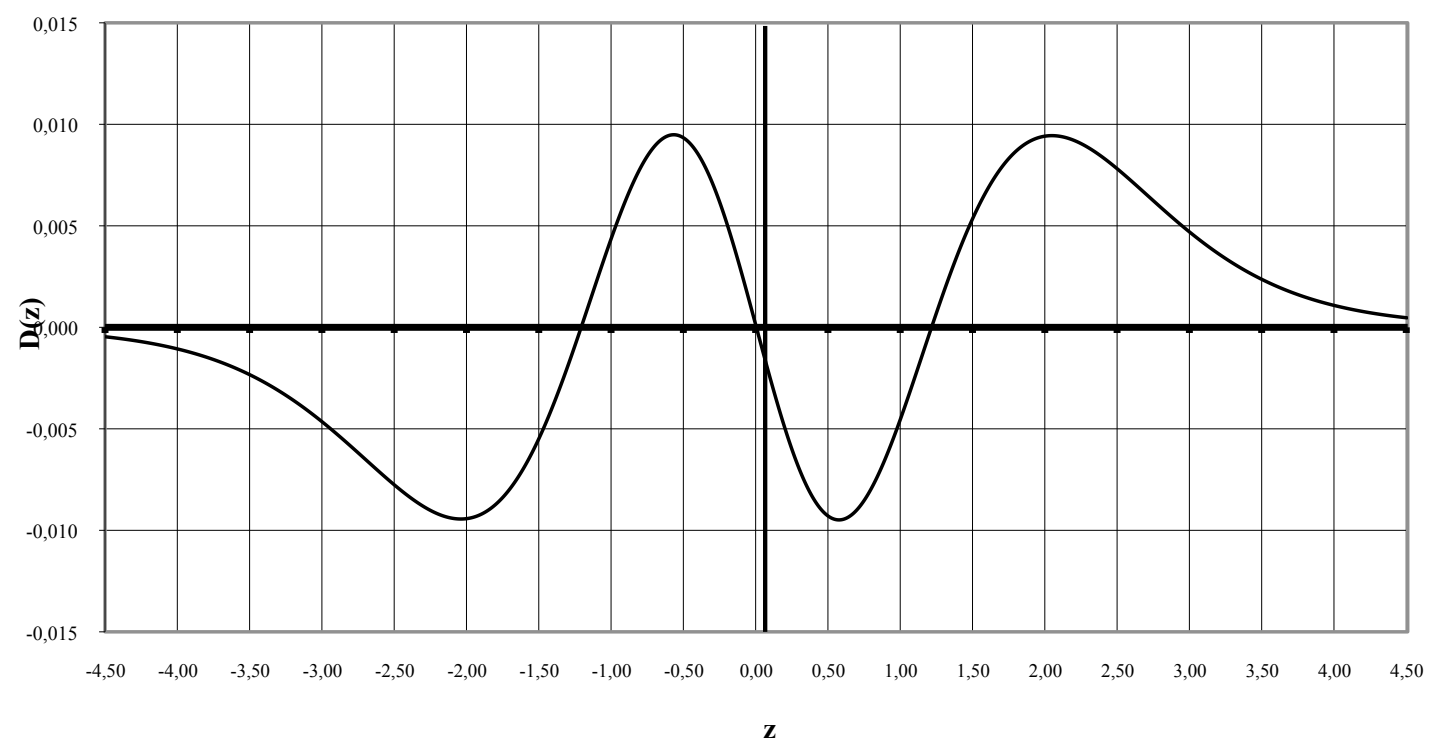

Figure 1. "The difference between the logistic function and cumulative normal distribution".

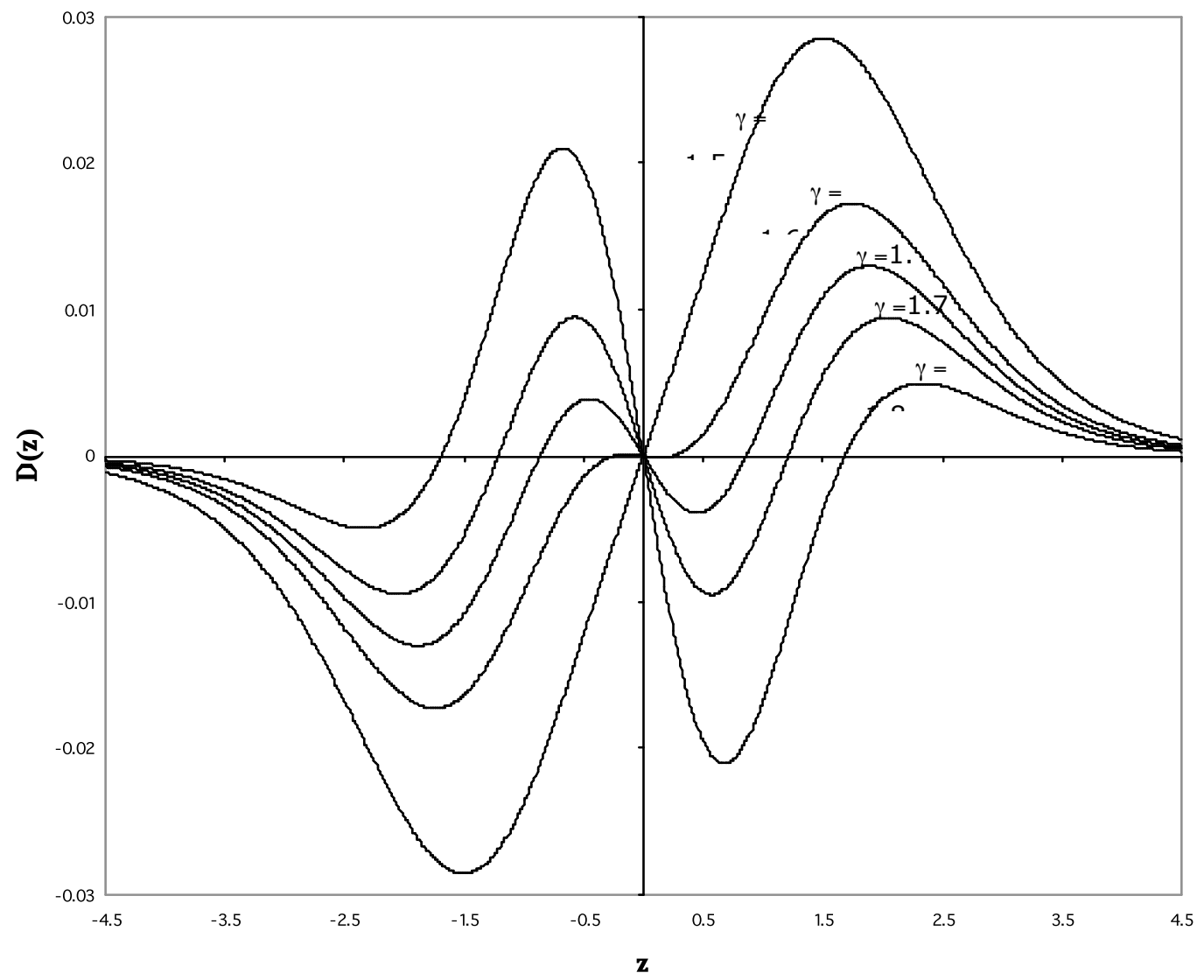

Figure 2. "The variation of the deviation function for different values of $\gamma^{\prime \prime}$. 


\subsection{A Polynomial Logistic Regression Function}

In order to address the first question, a functional relationship between $\gamma$ values and their corresponding non-origin intersection points on the $x$-axis, $\hat{\gamma}(x)$, is established, as shown in Figure 3. This is to ensure that the effect of $\gamma$ on the absolute deviations is better captured. In this particular case, a second-order regression function turns out to be an appropriate function for $\hat{\gamma}(z)$. For example, a polynomial regression without considering the absolute deviations is obtained as $\hat{\gamma}(z)=0.061 z^{2}+1.622$, as shown in Figure 3 . Based on this experimental observation, $\hat{\gamma}(z)=\gamma_{1} z^{2}+\gamma_{2}$. Notice that the equation does not include the firstorder $z$ term. This is due to the fact that the function is symmetric about the $y$ axis. By incorporating the standard normal distribution function, the modified absolute deviation function is then given by:

$$
D(z)=\left|\int_{-\infty}^{z} \frac{1}{\sqrt{2 \pi}} \exp \left[-\frac{y^{2}}{2}\right] d y-\frac{1}{1+\exp \left[-\left\{\gamma_{1} z^{3}+\gamma_{2} z\right\}\right]}\right|
$$

Equation 9. "Modified Absolute Deviation Function".

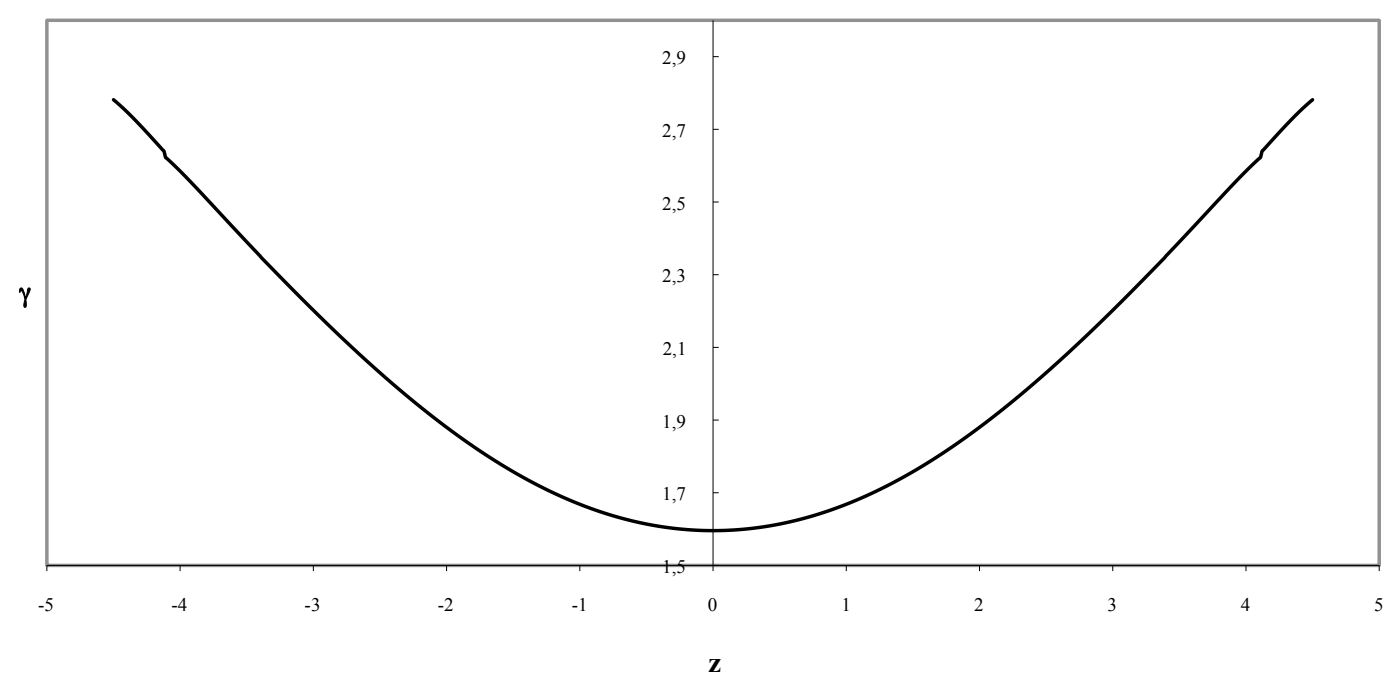

Figure 3. "The variation of $\gamma$ as a function of $z$ ". 


\subsection{Minimax Decision Criterion}

In order to address the second question raised in section 3.2 and to make the analysis on a consistent basis, the minimax decision criterion, which minimizes the maximum deviation, is employed. The minimax criterion is widely used in decision theory. Using the generalized reduced gradient algorithm, the parameters $\gamma_{1}$ and $\gamma_{2}$ are determined by minimizing the maximum deviation using equation 9 . As a result, the minimum of the maximum deviation with 0.00014 occurs at $z= \pm 3.16$ for $\gamma_{1}=0.07056$ and $\gamma_{2}=1.5976$. Therefore, the following equation gives the best approximation to the standard cumulative normal distribution, while minimizing the maximum deviation:

$$
F(z) \approx \frac{1}{1+e^{-\left(0.07056^{*} z^{3}+1.5976 * z\right)}}
$$

Equation 10. "Best Fit Two Parameter Logistic Function".

\section{Accuracy of the Proposed Logistic Approximation}

As it is the case for any approximate function, the proposed model should be evaluated in order to ensure that it is an appropriate approximation in terms of accuracy and range of validity. Table 2 compares the proposed logistic approximation with two popular approximations developed by Hamaker (1978) and Lin (1988), as well as the cumulative normal distribution. As it can be clearly seen, Hamaker's and Lin's approximations are valid for only one half of the cumulative normal distribution (i.e., the tail probabilities). Figure 4 illustrates the deviation between the cumulative standard normal distribution, and the approximate functions. The proposed approximation is more accurate when $-2.2<x<+2.2$. The proposed approximate function is validated by comparing it to the actual standard normal distribution table in order to check the accuracy and range of validity. As shown in Table 3, the accuracy is quite high. Most of the probabilities using the proposed approximation coincide to the probabilities shown in the standard normal table. The maximum error is less than $1.4 \times 10^{-4}$. It is also noted that the approximate function is relatively simple, compared to others available in the literature. 


\begin{tabular}{|c|c|c|c|c|}
\hline $\mathbf{Z}$ & Standard Normal & Hamaker (1978) & Lin (1988) & Proposed \\
\hline-3.00 & 0.001349 & 0.001337 & 0.001339 & 0.001232 \\
\hline-2.50 & 0.006209 & 0.006201 & 0.006203 & 0.006081 \\
\hline-2.00 & 0.022750 & 0.022869 & 0.022848 & 0.022761 \\
\hline-1.50 & 0.066807 & 0.067166 & 0.067044 & 0.066948 \\
\hline-1.00 & 0.158655 & 0.158855 & 0.158556 & 0.15867 \\
\hline-0.50 & 0.308537 & 0.308018 & 0.307661 & 0.308398 \\
\hline 0.00 & 0.500000 & 0.500000 & 0.500000 & 0.500000 \\
\hline 0.50 & 0.691462 & 0.691982 & 0.692338 & 0.691602 \\
\hline 1.00 & 0.841344 & 0.841145 & 0.841443 & 0.84133 \\
\hline 1.50 & 0.933192 & 0.932834 & 0.932955 & 0.933052 \\
\hline 2.00 & 0.977249 & 0.977131 & 0.977151 & 0.977239 \\
\hline 2.50 & 0.993790 & 0.993799 & 0.993796 & 0.993919 \\
\hline 3.00 & 0.998650 & 0.998663 & 0.998660 & 0.998768 \\
\hline
\end{tabular}

Table 2. "Illustration of the proposed logistic approximation vs. the cumulative normal distribution and that of Hamaker (1978) and Lin (1988)".

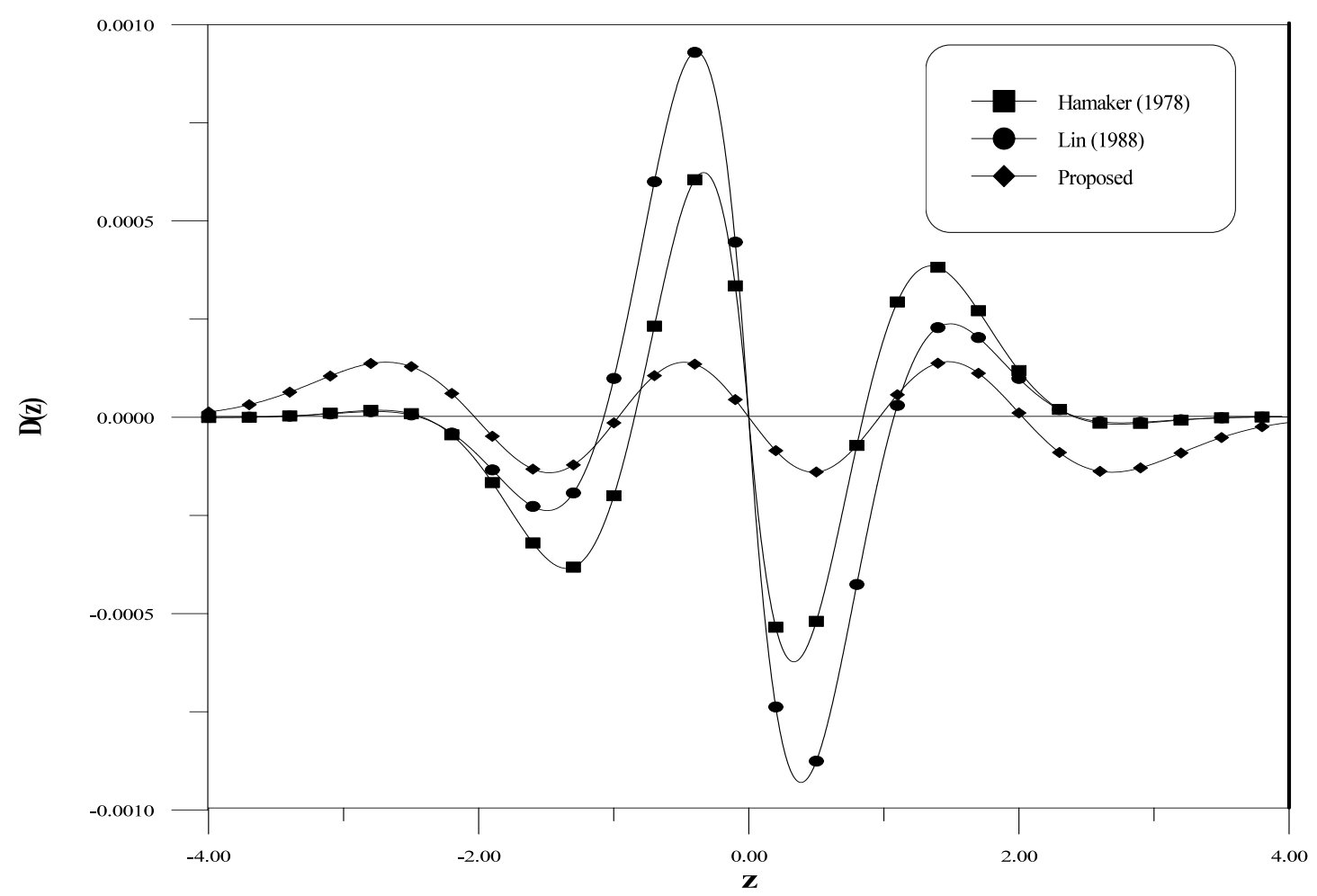

Figure 4. "Graphical illustration of the deviation of the proposed logistic approximation, Hamaker's (1978), and Lin's (1988) to the cumulative standard normal distribution". 


\begin{tabular}{|c|c|c|c|c|c|c|c|}
\hline & 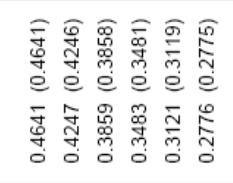 & 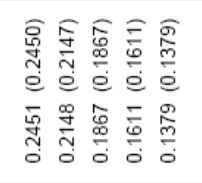 & 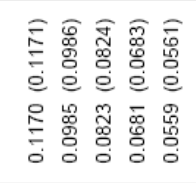 & 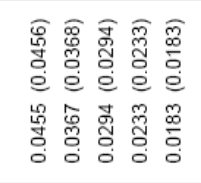 & 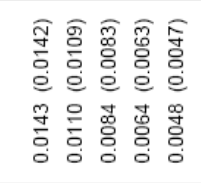 & 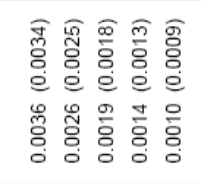 & $\begin{array}{ll} & 0 \\
0 & 0 \\
0\end{array}$ \\
\hline & 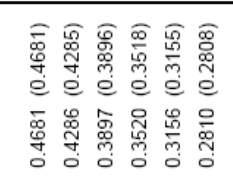 & 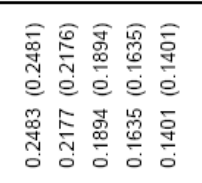 & 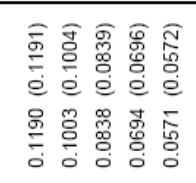 & 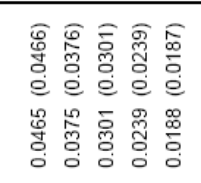 & 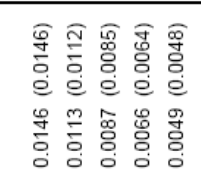 & 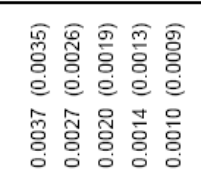 & 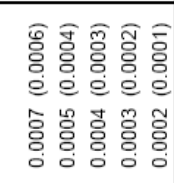 \\
\hline & 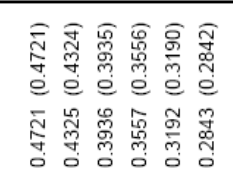 & 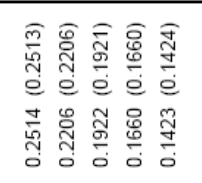 & 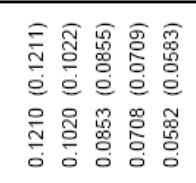 & 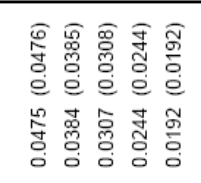 & 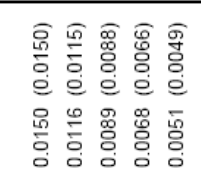 & 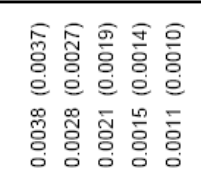 & $\begin{array}{l}0.0 \\
0.0 \\
0 \\
0 \\
0 \\
0\end{array}$ \\
\hline & 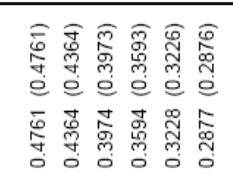 & 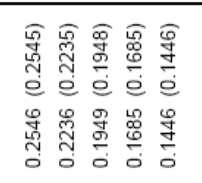 & 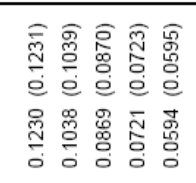 & 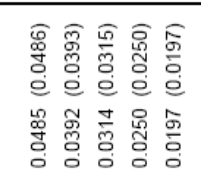 & 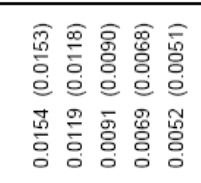 & 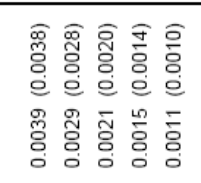 & \\
\hline & 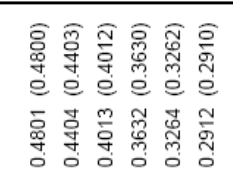 & 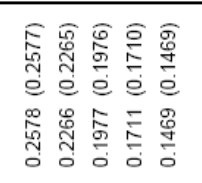 & 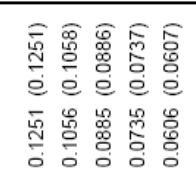 & 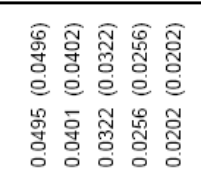 & 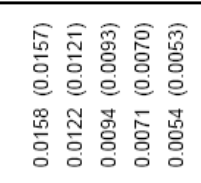 & 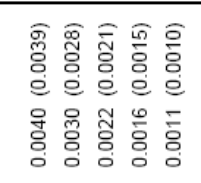 & \\
\hline & 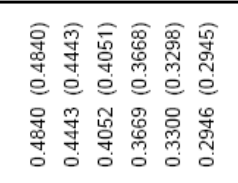 & 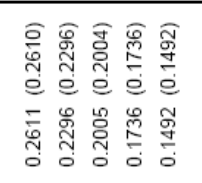 & 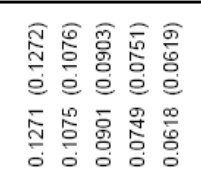 & 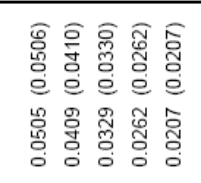 & 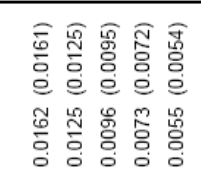 & 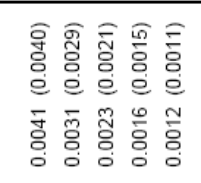 & \\
\hline & 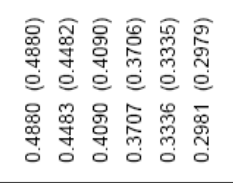 & 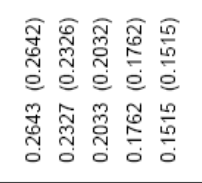 & 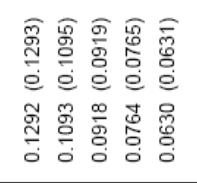 & 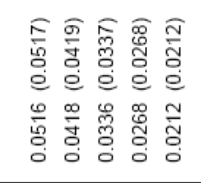 & 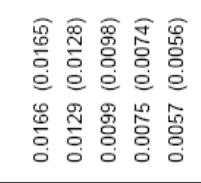 & 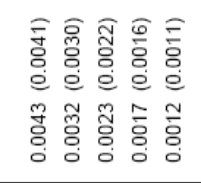 & 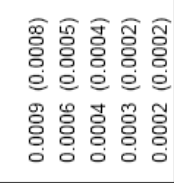 \\
\hline & 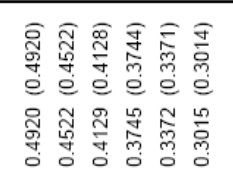 & 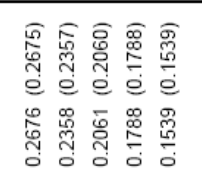 & 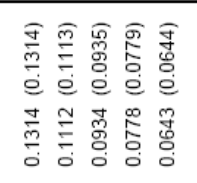 & 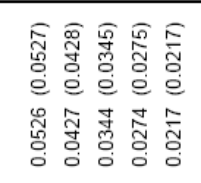 & 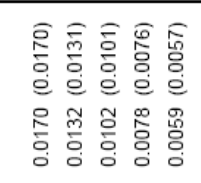 & 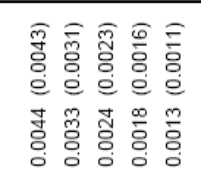 & \\
\hline & 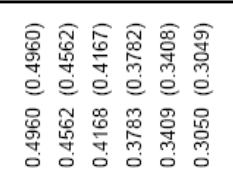 & 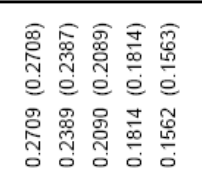 & 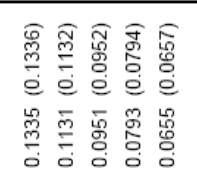 & 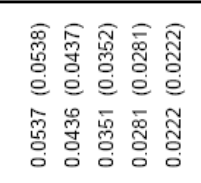 & 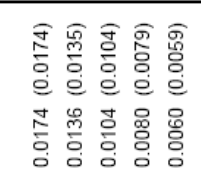 & 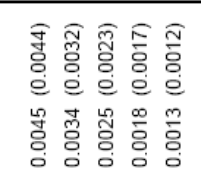 & \\
\hline & 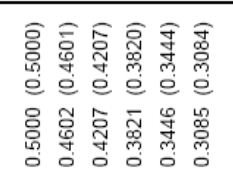 & 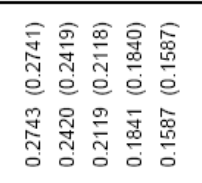 & 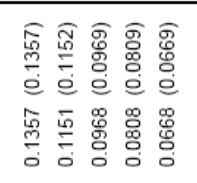 & 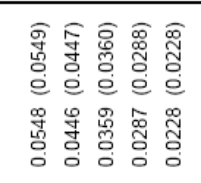 & 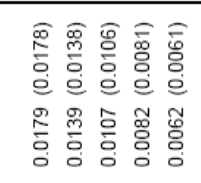 & 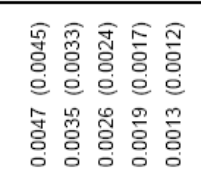 & \\
\hline & 응웅 궁융영 & $\circ 0$ & $\stackrel{\circ}{\circ}$ & : 욤 & $\stackrel{\leftrightarrow}{\leftrightarrow}$ & & \\
\hline
\end{tabular}

Table 3. "Standard Normal Table vs. Proposed Approximation (Approximation Values)". 


\section{References}

Cadwell, J. H. (1951). The Bivariate Normal Integral. Biometrika, 38, 475-479.

Hamaker, H. C. (1978). Approximating the Cumulative Normal Distribution and its Inverse. Applied Statistics, 27, 76-77.

Hart, R. G. A. (1963). Close Approximation Related to the Error Function. Mathematics of Computation, 20, 600-602.

Hiller, F. S., \& Liberman, G. J. (2001). Introduction to Operations Research. $7^{\text {th }}$ Ed., New York: McGraw-Hill.

Hoyt, J. P. A. (1968). Simple Approximation to the Standard Normal Probability Density Function. American Statistician, 22(3), 25-26.

Johnson, N. L., \& Kotz, S. (1969). Distributions in Statistics: Continuous Univariate Distributions. Houghton Mifflin Company: Boston.

Lin, J. T. (1988). Alternative to Hamaker's Approximations to the Cumulative Normal Distribution and its Inverse. Applied Statistics, 37, 413-414.

Lin, J. T. (1989). Approximating the Normal Tail Probability and its Inverse for use on a Pocket-Calculator. Applied Statistics, 38, 69-70.

Lin, J. T. (1990). A Simpler Logistic Approximation to the Normal Tail Probability and its Inverse. Applied Statistics, 39, 255-257.

Polya, G. (1945). Remarks on Computing the Probability Integral in One and Two Dimensions. Proceedings of the $1^{\text {st }}$ Berkeley Symposium on Mathematical Statistics and Probability, 63-78.

Schucany, W. R.. \& Gray, H. L. (1968). A New Approximation Related to the Error Function. Mathematics of Computation, 22, 201-202.

Zelen, M., \& Severo, N. C. (1966). Probability Functions. In Handbook of Mathematical Functions, Ed. Abramowitz and I. A. Stegun, Washington D.C.: Department of US Government. 
Article's contents are provided on a Attribution-Non Commercial 3.0 Creative commons license. Readers are allowed to copy, distribute and communicate article's contents, provided the author's and Journal of Industrial Engineering and Management's names are included. It must not be used for commercial purposes. To see the complete license contents, please visit http://creativecommons.org/licenses/by-nc/3.0/. 